\title{
Improving Health Equity: The Promising Role of Community Health Workers in Canada
}

\section{Amélioration de l'égalité en matière de santé : le rôle prometteur des travailleurs en santé communautaire au Canada}

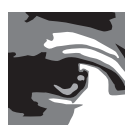

SARA TORRES, PHD

Postdoctoral Fellow, Institut de recherche en santé publique de l'Université de Montréal (IRSPUM)

Montreal, QC

RONALD LABONTÉ, PHD, FCAHS

Canada Research Chair, Globalization and Health Equity

Professor, University of Ottawa, ON

Professor, Flinders University of South Australia

Adjunct Professor, University of Saskatchewan, SK

DENISE L. SPITZER, PHD

Canada Research Chair in Gender, Migration and Health Associate Professor, Institute of Feminist and Gender Studies

University of Ottawa

Ottawa, ON

CAROLINE ANDREW, PHD

Faculty of Social Sciences, University of Ottawa

Ottawa, ON

CAROL AMARATUNGA, PHD

Adjunct Professor, School of Public Health and Social Policy

University of Victoria

Victoria, BC 


\begin{abstract}
This article reports findings from an applied case study of collaboration between a community-based organization staffed by community health workers/multicultural health brokers (CHWs/MCHBs) serving immigrants and refugees and a local public health unit in Alberta, Canada. In this study, we explored the challenges, successes and unrealized potential of $\mathrm{CHWs} / \mathrm{MCHBs}$ in facilitating culturally responsive access to healthcare and other social services for new immigrants and refugees. We suggest that health equity for marginalized populations such as new immigrants and refugees could be improved by increasing the role of CHWs in population health programs in Canada. Furthermore, we propose that recognition by health and social care agencies and institutions of CHWs/MCHBs, and the role they play in such programs, has the potential to transform the way we deliver healthcare services and address health equity challenges. Such recognition would also benefit CHWs and the populations they serve.
\end{abstract}

\title{
Résumé
}

Cet article fait état des résultats d'une étude de cas sur la collaboration entre une organisation communautaire qui emploie des travailleurs en santé communautaire (TSC) et des courtiers en santé multiculturelle (CSM) pour desservir des populations immigrantes et de réfugiées ainsi qu'une unité locale de santé publique en Alberta, Canada. Dans cette étude, nous avons exploré les défis, les succès et le potentiel non réalisé des TSC et des CSM pour faciliter l'accès aux services de santé et autres services sociaux par les nouveaux immigrants et les réfugiés. Nous estimons qu'il est possible d'améliorer l'égalité en matière de santé pour les populations marginalisées telles que les nouveaux immigrants et les réfugiés, et ce, en accroissant le rôle des TSC dans les programmes liés à la santé des populations au Canada. De plus, nous croyons que si les agences et institutions de santé et de services sociaux reconnaissent les TSC et les CSM, ainsi que leur rôle dans de tels programmes, cela pourrait transformer la façon de fournir les services de santé et de traiter les défis en matière d'égalité. Une telle reconnaissance serait aussi avantageuse pour les TSC et les populations qu' ils desservent.

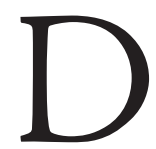

espite the virtual universality of Canada's healthcare system,

marginalized populations, such as immigrants and refugees, continue to experience barriers to accessing the health system (Beiser 2005; Pottie et al. 2008; Pottie et al. 2011; Spitzer 2004). Various approaches to improving access are currently used by local healthcare institutions, for example, interpreters and patient navigators, or culture-specific programs (Black et al. 2006). In this paper, we examine a particular model of collaboration between a community health worker $(\mathrm{CHW}) /$ multicultural health broker (MCHB) initiative and a local public health unit focusing on perinatal health. Although more community outreach collaborations have been called for as a way to meet unaddressed needs (Green et al. 2013), little attention has been given to existing collaborations between "communities on the margin" and formal public healthcare systems. This paper adds to the emerging literature on 
an independent $\mathrm{CHW} / \mathrm{MCHB}$ model that provides culturally responsive access to healthcare services for new immigrant and refugee populations in Canada (Black et al. 2006; Meyer et al. 2003; Torres 2013). We also discuss the role of CHWs/MCHBs in addressing needs linked to social determinants of health.

We begin with an overview of $\mathrm{CHW}$ s/MCHBs in Canada. Thereafter, we describe the methods used in our study and present selected research results. We conclude with a discussion of the implications of the findings for more effective incorporation of $\mathrm{CHWs} / \mathrm{MCHBs}$ within Canadian health systems.

\section{First author's statement}

The first author (ST) is an immigrant with past experience as a CHW. She has been actively involved in conceptualizing and implementing CHW programs for immigrant communities, in particular several initiatives targeting Hispanic populations in Canada. These experiences led her to the study reported herein, and a concern that a lack of formal recognition by health systems of the roles played by CHWs in population health programs not only limited their potential effectiveness in improving the health of marginalized populations, but also marginalized CHWs themselves.

\section{Overview of CHWs}

In this paper we use the term community health worker when referring to generic community outreach programs or practices delivered by front-line health workers who are members of the communities they serve. CHWs are considered to have a deep understanding of the issues faced by these communities in accessing health and social services, and are able to offer linguistically and culturally appropriate assistance.

Recent literature indicates that CHWs who share a similar cultural background with immigrants and refugees are better able to respond to the health needs of these populations (Wells et al. 2011). Similarly, literature on cultural brokering (bridging or mediating between different cultural groups to effect change) highlights the role of CHWs as cultural brokers in increasing access to, and enhancing the delivery of, culturally competent care (National Center for Cultural Competence 2004). Cultural brokering is an important concept in the study we report on, as it is the approach espoused by the Health Co-Op that constitutes our case (Ortiz 2003). The Health Co-Op adapted cultural brokering theory (Jezewski 1995) and developed their multicultural health brokering approach. The "cultural responsiveness" of CHWs has been an important component of their practice for more than five decades in low-, middle- and high-income countries, enabling them to address a variety of health issues (Andrews et al. 2004; Lewin et al. 2005). CHWs in high-income countries such as Canada often work in traditionally underserved areas or in communities experiencing vulnerability, frequently requiring cross-cultural competencies (Clark and Surry 2007; Hodnett 2003; Khanchandani and Gillam 1999; Rhodes et al. 2007; Swider 2002).

CHWs are mainly unlicensed professionals (whether paid or unpaid) with varying levels of academic or non-academic training. In Canada, as in many other countries, there is no 
official coordination among institutions and organizations on the conceptualization, implementation and delivery of CHW training programs. This means that most health system institutions - public health units, community health centres and other community-based organizations - develop their training and curriculum independently.

\section{CHWs and healthcare systems}

$\mathrm{CHWs}$ can be linked to healthcare systems in one of two ways: either working directly within the formal system or operating independently. In either model, CHW' abilities to reduce barriers to access to health services depend on their relationship to primary care or public health institutions (e.g., community health centres, hospitals, public health units and regional health authorities). Canadian evidence on these relationships (how they are organized and managed, and the range of activities supported) is slowly emerging in academic literature (Black et al. 2006; Meyer et al. 2003), but often remains located in grey literature (Black et al. 2007; Chiu et al. 2009; Pottie et al. 2008).

International studies reveal a variety of organizational and financial arrangements governing the use of CHWs. In countries like Brazil and Iran, CHW programs are publicly funded, with CHWs fully integrated within the formal health system. The size of the workforce is considerable in both of these countries, with Brazil employing 257,265 (Johnson et al. 2013) and Iran 31,000 (Javanparast et al. 2011) workers. CHWs in the US, estimated to number more than 121,000 (HRSA 2007), are recognized as part of the health workforce and have their own CHW Standard Occupational Category - SOC \#21 - 1094 Community Health Worker - which recognizes these workers as a distinct occupation (Balcázar et al. 2011; Federal Register 2009).

In Canada, Australia and New Zealand, CHWs are not systematically integrated within government-funded universal healthcare systems, and there are little data on the size of this workforce.

Canadian CHWs had been disconnected from each other, but have recently formed the Community Health Worker Network of Canada - the first organization of its kind in the country. Among the concerns discussed by network members include the lack of recognition and the unregulated status of CHWs; the need to organize the workforce at the local, regional, provincial and national levels; the search for a single definition for their work; the need to conduct research on the workforce's composition and competencies; and the potential for adopting a set curriculum or standardized training for CHWs. Network members are also reflecting on how the two CHW practice models (independent or integrated, described below) affect their capacity to remove access barriers to health and other services in marginalized communities, or to serve as cultural brokers.

\section{INDEPENDENT MODEL}

In this model, independent organizations, often established by CHWs themselves, offer 
services that target marginalized communities. These organizations and the CHWs working within them obtain contract funding to deliver their services independently of public healthcare institutions, but often in collaboration with them. When funded, organizations retain complete autonomy in the work's conceptualization and programming. The wages and hours to deliver the services are not equivalent to those of $\mathrm{CHWs}$, who may be working within the formal healthcare system; typically, funding allows for a mixture of part-time and full-time CHW employment. The Multicultural Health Brokers Co-Operative (hereafter called the Health Co-Op) and MCHBs in Edmonton fall within an independent CHW program model.

Independent models are marked by high levels of health promotion activity, as distinct from a focus on specific service delivery. The main health system's concern with independent models is that they may have less accountability and supervision, and that the CHWs may have less training, than CHW program delivery through integrated models. Furthermore, given the lack of resources, it may also be more difficult to evaluate service outcomes in independent models.

\section{INTEGRATED MODEL}

In this model, CHWs are staff within public health or primary care institutions, which have as part of their mandate the delivery of programs targeting populations experiencing marginalization. The CHW programs in Brazil and Iran are examples of highly integrated models. In Canada, the Women's Health Educators, CHWs who work within Hamilton's Ontario Public Health division (Black et al., 2007), are an example of an integrated model. These workers are usually well-paid with good benefits, as well as reasonable work hours and caseloads, which is often not the case for CHWs operating in independent models. Generally, CHWs and their professional allies in public health units reach out to marginalized communities with specific programs and key health messages. In situations of pandemics, they orchestrate campaigns to ensure that contagious diseases do not spread among these populations. Other Canadian examples of integrated models include the Multicultural Health Team operating under Ottawa Public Health and the Toronto Public Health Unit Peer Nutrition workers who reach out to immigrant and racialized communities. One concern with the integrated model is that its responsiveness to marginalized community concerns may be constrained by requirements for bureaucratic approval that is often needed for new initiatives. Another concern with this model is that CHWs are more and more focused around fulfilling technical activities and practices traditionally performed with the scope of health services rather than focused on intersectoral actions, community development and social participation (Rodrigues Fausto et al. 2011).

\section{Methods}

Data for this paper are a subset of a case study conducted for the first author's doctoral research. The investigation applied an instrumental case study design (Stake 1995) to examine issues that went beyond the case itself (Stake 2006). 
The study analyzed two sub-programs of the Health Co-Op as embedded mini-cases (Yin 2003): the Perinatal Outreach program, funded by Alberta Health Services (AHS), and the Health for Two program, funded by the Canada Prenatal Nutrition Program and AHS. Finally, the study examined the MCHBs' practice to understand their role as part of a health human resource workforce that operates independently of the healthcare system. When discussing the Health Co-Op workers, we use the term MCHBs, as this is how they refer to themselves. The case study triangulated multiple research strategies and data sources, including participant and direct observation, in-depth interviews, document review and analysis of policy and other documents and quantitative analysis of the MCHBs' client caseload database $(\mathrm{n}=3,442)$. Data also included descriptive and analytical field notes taken throughout the course of the onsite research. This paper centres on the analysis of 23 of the 44 qualitative interviews conducted for the study (16 of these interviewees were MCHBs working for the Health Co-Op and seven were health professionals within AHS), as well as data from the MCHBs' client caseload database. Interviews were conducted between July 2009 and May 2010. The database included caseloads of $46 \mathrm{MCHBs}$ (and some supervisors or mentors) for the 12-month period between September 2009 and September 2010 (MCHB Co-op 2010b). Depending on the program, an MCHB case was defined as a mother with a child or children 0 to 6 years old, a family with a child with a disability, the parents whose children are experiencing abuse or neglect, or a senior experiencing isolation (see Figure 1). Hand-coding and the qualitative analysis software QSR Nvivo8 were used in coding and data management. Inductive analysis was conducted for the interviews, and descriptive analysis was performed on the MCHBs' client caseload database.

FIGURE 1. MCHB client caseload

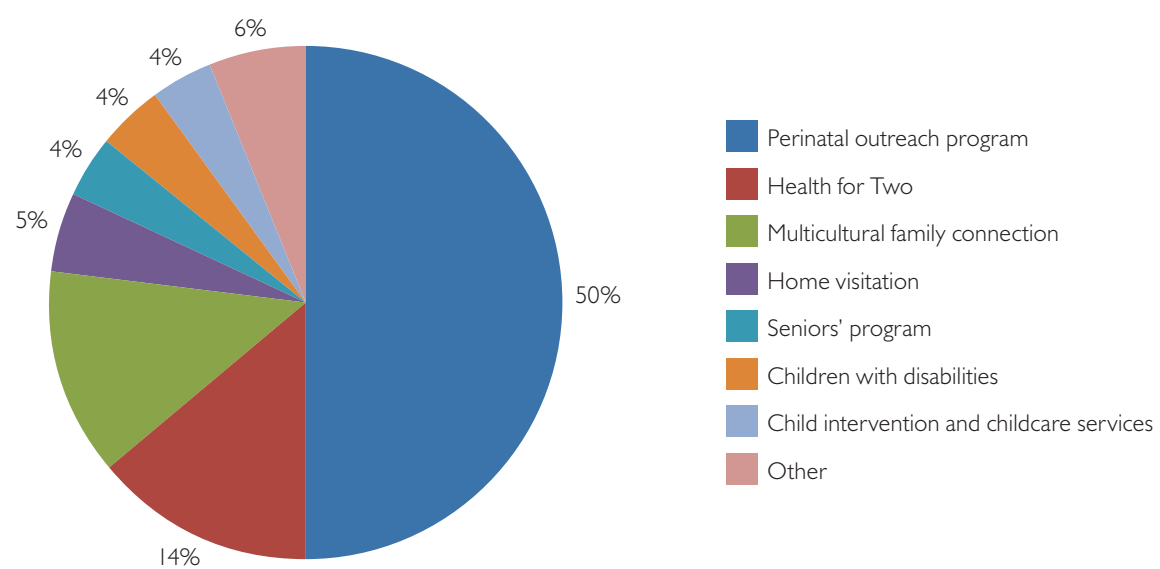

Source: Torres, S. 2013: 112. 


\section{Findings}

\section{The bealth $\mathrm{Co}-\mathrm{O} p$ and $\mathrm{MCHBs}$}

The Health Co-Op's independently run programming has been offering culturally responsive services ever since its founding in 1998. The Health Co-Op's 54 MCHBs offer an important contribution to public health workforce development and a unique Canadian CHW model. $\mathrm{MCHBs}$ provide culturally and linguistically appropriate services to immigrant and refugee families from at least 18 different cultural backgrounds, and collectively are able to speak 29 languages (MCHB Co-op 2004a).

One MCHB explained the need to provide services that are culturally relevant to women, even if that includes bringing "cultural food" to a new mother in the hospital:

Yeah. Sometimes, like, you know, she had a baby at 6 oclock ... at night, or 7, or 8, 9, 10 o'clock at night ... she has nobody, she has no language ... she's in the hospital, and she's calling you.... Of course, you have to take certain food from your country, ... because she's not going to eat the food that they're giving her ... So there are certain things you go out of your way because you know what it is like.... When they call you it is because they are desperate in that time, so some things ... you have to do.

MCHBs do home visits to pregnant women and new mothers, accompany patients to doctors' appointments, take mothers and children to vaccination clinics and accompany women during child labour and delivery. $\mathrm{MCHBs}$ also do group work, undertake community development initiatives and provide educational outreach on chronic disease prevention and management, as well as offer family intervention programs to address child abuse and neglect. MCHBs work independently of health and social service government ministries while being funded by them; the AHS Edmonton Zone has funded MCHBs for the past 15 years. Under the 2009-2010 Perinatal Outreach service contract, the Health Co-Op received just over $\$ 300,000$ dollars from AHS' Maternal Child funding envelope to serve 2,340 families, representing approximately $10 \%$ of Edmonton families with births (MCHB Co-op 2010b). Pursuant to this agreement, MCHBs received training by AHS public health nurses on issues related to prenatal and postnatal health. As an independently run Health $\mathrm{Co}-\mathrm{Op}, \mathrm{MCHBs}$ control and manage their time to meet the needs of their clients, sometimes making themselves available to help clients in the evenings or on weekends, which mainstream health service providers typically do not do. These types of services, however, are not fully financed by AHS, and MCHBs often use their "own time," as they describe it, to support immigrant and refugee women and their families, effectively subsidizing the publicly funded program. Indeed, being available to support mothers in times of need is what often makes it possible for MCHBs to do their perinatal work. As one MCHB stated: 
... let's say Saturday one crisis comes, ... or evenings, ... I have to go to the hospital, mom is in labour ... she needs someone, she has no one there, I can't say it's not my working hours, so I stay there, you know, long time so!

MCHBs also assist families with other needs linked to social determinants of health, including helping families to fill in forms for the child tax credit, schooling, employment insurance or undertaking child intervention/protection work.

Figure 1 illustrates the MCHBs' caseload, including health and other related programs. While the Perinatal and Health for Two programs funded by AHS occupy $64 \%$ of the workers' reported caseload, the child intervention (or child protection) program uses only $4 \%$ (MCHB Co-op 2010a). In real terms, however, the latter program is more demanding because victims of family violence require intense support over a long period. $\mathrm{MCHBs}$ who work in violence prevention and child intervention receive training in these areas from Alberta Children Services to make sure that MCHBs are aware of their legislative responsibilities as workers.

Finally, $\mathrm{MCHBs}$ developed their own multicultural health brokering theory to guide their practice (Ortiz 2003). Multicultural health brokering is a relationship-based practice based on trust and confidence established between $\mathrm{MCHBs}$ and the women and communities they help (Torres 2013).

The practice standards of MCHBs daily activities embody both the principles and the values of health brokering theory (Ortiz 2003). These standards are:

... specific guidelines to guide Multicultural Health Brokers in their day-to-day practice. The practice standards are codes of conduct that will help support decisions and actions of the worker in an ethical and appropriate manner (MCHB Co-Op 2004b:8).

They are based on a dedication to empowering people and communities; are rooted in the wisdom, knowledge and culture ways of people; and are based on principles of social justice, equity and democracy (MCHB Co-op 2004b). The worker Health Co-Op structure facilitates the MCHBs' ability to follow and advocate for these principles.

MCHB practice is unlike other CHW models in Canada because MCHBs do not focus only on service delivery and community development, but also engage with communities in their role as health brokers.

$\mathrm{MCHB}$ interviews revealed their commitment to responding to health and social needs of the communities, whether or not the Health $\mathrm{Co}-\mathrm{Op}$ received funding to service those needs. $\mathrm{MCHBs}$ viewed this situation as reflecting a growing gap in access to health and social services, which would require multi-ministry and multi-stakeholder support to address. 


\section{Perspectives from bealth professionals}

Alberta's health system has undergone several rounds of restructuring in the past two decades, although the funding for the MCHB program has been maintained. All AHS health professionals in the study indicated that the MCHB Perinatal Outreach program was valuable. Firstly, MCHBs helped the health unit to meet its mandate to serve immigrants and refugees, and the health unit's staff and managers to learn about immigrant and refugee communities from the MCHBs.

One health professional stated:

Well, I don't think we could do our work without them ... I think that they're hugely influential in the outcomes ... I mean certainly there's a role for both of us [public health] within helping that family with health outcomes, but ... the brokers ... we really depend on them.

Secondly, MCHBs understood different cultures, provided culturally appropriate services and were able to help clients in different areas:

I'm completely convinced that every single newcomer to the country should have a health broker, I mean, I have no doubts about that ... immigrant families manage fairly well in comparison to the refugee families, but the refugee families really struggle and should, do need that kind of support, I feel quite strongly.

Thirdly, MCHBs have helped most health services providers in the city to understand the need to look at "culture" when serving immigrants and refugees. One professional explained:

... And like I said it's not just about the language, it's about the whole cultural appropriateness and navigating the system.

Finally, MCHBs are not interpreters, they are cultural brokers. When they accompany patients to medical appointments, they help health professionals to understand clients' issues better, especially when the client does not speak English fluently. One health professional referred to MCHBs' contributions to the health system as "saving lives."

Most health professionals thought that the health system was responding well to the perinatal care needs of existing immigrant and refugee communities, but was not providing adequate funding to provide for new cultural groups arriving in the city. Consequently, the Health Co-Op has had to divide funding received from AHS Edmonton Zone to serve more communities. This means that, owing to the high demands on MCHBs' time and the lack of funding to support their work, MCHBs are at "maximum capacity." AHS health professionals also acknowledge that MCHBs' wages are not at the same level as other staff in the system doing similar jobs. 


\section{Discussion}

The collaboration between an independent MCHB model and AHS' public health unit in Edmonton reveals the complementary role that MCHBs can play in meeting the health system's mandate to reduce barriers to marginalized populations' access to services. The MCHB model illustrates the importance of addressing health needs of immigrants and refugees through culturally and linguistically appropriate services that target issues linked to social determinants of health.

This article focused on the uniqueness of the MCHB Edmonton model because of its relevance to other cities and provinces in Canada that may wish to improve access to health and other services by working with CHWs operating independently of the formal healthcare system. We believe, however, that both independent and integrated CHW models have a role to play in improving the effectiveness of the Canadian health systems. Understanding and recognizing the different models in which $\mathrm{CHWs} / \mathrm{MCHBs}$ contribute to the health system is important for three reasons: these workers are part of the health human resources workforce; these workers target many populations (e.g., immigrants, Aboriginal people, women and children, people living on low income); and they foster collaboration between communities in the margin and public and primary care institutions, which can potentially reduce cost by improving health outcomes for members of these communities.

From a public policy perspective, and using the Province of Ontario as an example, the public health standards of the Ministry of Health and Long Term Care (MOHLTC 2008) could explore how both the $\mathrm{CHW} / \mathrm{MCHB}$ independent and integrated models can help in meeting the provincial standards governing boards of health. These standards are minimal criteria to ensure that barriers to accessing public health programs and services, such as language, economic circumstances and discrimination (MOHLTC 2008), are reduced. Barriers can include, but are not limited to, lack of education, low literacy levels, language, cultural differences, geography, economic circumstances, discrimination (e.g., age, sexual orientation, race), social factors (e.g., social isolation) and mental and physical disability (MOHLTC 2008). $\mathrm{CHWs} / \mathrm{MCHBs}$ working in a health brokering capacity are able to address several of these barriers.

$\mathrm{CHW}$ s/MCHBs are also well-placed to identify emerging needs among communities and to aid the health system in preventing families from falling through service gaps.

Undervaluing $\mathrm{CHWs}$ /MCHBs' work, however, can result in reduced uptake of services, compelling marginalized populations to delay seeking care or to present at emergency departments (Khandor et al. 2011), resulting in higher costs for the healthcare system (Crighton et al. 2007). By implication, federal, provincial and territorial health ministries should consider formal recognition of $\mathrm{CHWs} / \mathrm{MCHBs}$ as part of the health workforce. This may involve multi-ministry investment of sufficient funding to cover all the work hours of $\mathrm{CHWs} / \mathrm{MCHBs}$ to fully deliver the required services, including the important support work (Armstrong and Armstrong 2010) CHWs/MCHBs provide to marginalized populations. 


\section{Conclusions}

This paper, based on empirical research, described the collaboration between MCHBs in Edmonton and the local public health unit's maternal child health area to remove barriers to accessing perinatal health for immigrant and refugee women and their families. It also illustrated the strategies used by MCHBs to address the complex realities of these families, owing to other needs linked to social determinants of health, and highlighted the linguistically and culturally appropriate nature of their work. Additionally, the paper advanced a case for the formal recognition of $\mathrm{CHWs} / \mathrm{MCHBs}$ working in both independent and integrated models as part of the health human resources workforce, although not recognized as such.

The collaboration model described in this paper and the linguistically and culturally appropriate nature of $\mathrm{MCHB}$ work are part of their commitment to building a new community of practice to address the perinatal health and other social needs of immigrant and refugee families (Torres et al. 2013). This community of practice is based on MCHBs' ability to articulate, reflect on and monitor their practice; to operate and sustain an independent organization; to develop and maintain and seek intersectoral, cross-governmental collaboration; and to develop programs that address the social determinants of health (Torres et al. 2013: 316).

$\mathrm{CHW} / \mathrm{MCHB}$ policy and programs represent an innovative approach and their adoption by other health systems in Canada could spearhead a new, transformative community health workforce.

As one of the first in-depth studies about CHWs/MCHBs in Canada, this work holds promise for shaping and influencing the rise of a new generation of evidence-informed population health programs in the country. Doing so will benefit the workers, the population they serve and the healthcare system.

\section{Acknowledgements}

We thank the participants at both the MCHB Co-op and AHS for sharing their knowledge and making it possible to complete this case study research.

Funding for this study was awarded by the Population Health Improvement Research Network (PHIRN) through funding from the Ontario Ministry of Health and Long Term Care, Funding Agency Ref. No. 06548, and by the CIHR-Institute of Gender and Health Doctoral Awards program.

Correspondence may be directed to: Sara Torres, $\mathrm{PhD}$, Postdoctoral Fellow, Institut de recherche en santé publique de l'Université de Montréal (IRSPUM)/Direction de Santé et des Services Sociaux de Montréal, Pavillon du Parc, 7101, ave. du Parc, 3064, Montréal, QC; tel.: 514-343-6143 ext. 5427; e-mail: sara.torres@alumni.uottawa.ca. 


\section{References}

Andrews, J.O., G. Felton, M.E. Wewers and J. Heath. 2004. "Use of Community Health Workers in Research with Ethnic Minority Women." Journal of Nursing Scholarship 36(4): 358-65.

Armstrong, P. and H. Armstrong. 2010. The Double Ghetto: Canadian Women and Their Segregated Work (3rd ed.). Don Mills: Oxford University Press.

Balcázar, H.G., L. Rosenthal, J.N. Brownstein, C.H. Rush, S. Matos and L. Hernandez. 2011. "Community Health Workers Can Be a Public Health Force for Change in the United States: Three Actions for a New Paradigm." American Journal of Public Health 101(12): 2199-203.

Beiser, M. 2005. "The Health of Immigrants and Refugees in Canada." Canadian Journal of Public Health 96(Suppl 2): $\mathrm{S} 30-44$.

Black, M., C. Campbell, B. Carpio, J. Crawford, A. Frisina, T. Hack et al. 2007. Women's Health Educators: Pilot Project: 2003-2005. Retrieved February 22, 2014. <http://www.hamilton.ca/HealthandSocialServices/ PublicHealth/Cancer/WomensHealthEducator.htm $>$.

Black, M., A. Frisina, T. Hack and B. Carpio. 2006. "Improving Early Detection of Breast and Cervical Cancer in Chinese and Vietnamese Immigrant Women." Oncology Nursing Forum 33(5): 873-76. doi: 10.1188/06.ONF.873876.

Chiu, Y., L. Ortiz and R. Wolfe. 2009. "Beyond Settlement Strengthening Immigrant Families, Communities and Canadian Society Through Cultural Brokering," In "Our Diverse Cities. Edmonton: Metropolis."

Clark, P.A. and L. Surry. 2007. "Mercy Health Promoters: A Paradigm for Implementing Third World Practices for Resource-Poor Conditions of the Developed World.” Medical Science Monitor 13(3): 1-8.

Crighton, E.J., S.J. Elliott, P. Kanaroglou, R. Moineddin and R.E. Upshur. 2007. “A Spatial Analysis of the Determinants of Pneumonia and Influenza Hospitalizations in Ontario (1992-2001)." Social Science E Medicine 64(8): 1636-50.

Federal Register. 2009. Part VI: Office of Management and Budget 2010 Standard Occupational Classification (SOC)_OMB's Final Decisions. Retrieved September 30, 2014. <http://www.bls.gov/soc/soc2010final.pdf>.

Green, M.E., E. Weir, W. Hogg, V. Etches, K. Moore, D. Hunter and R. Birtwhistle. 2013. “Improving Collaboration between Public Health and Family Health Teams in Ontario." Healthcare Policy 8(3): e93-104.

Hodnett E.D., S. Fredericks and F.S. Weston. 2003. “Support During Pregnancy for Women at Increased Risk of Low Birthweight Babies (Review)." The Cochrane Library (3). doi: 10.1002/14651858.CD000198.

HRSA. 2007. Community Health Worker National Workforce Study. Retrieved February 22, 2014. <http://bhpr. hrsa.gov/healthworkforce/reports/chwstudy2007.pdf>.

Javanparast, S., F. Baum, R. Labonté, D. Sanders, G. Heidari and S. Rezaie. 2011. "A Policy Review of the Community Health Worker Programme in Iran." Journal of Public Health Policy 32(2): 263-76. doi: 10.1057/ jphp.2011.7.

Jezewski, M.A. 1995. "Evolution of a Grounded Theory”. Advances in Nursing Science 17(3): 14-30.

Johnson, C.D., J. Noyes, A. Haines, K. Thomas, C. Stockport, A. Neves Ribas and M. Harris. 2013. "Learning from the Brazilian Community Health Worker Model in North Wales." Globalization and Health 9(25). doi:10.1186/1744-8603-9-25.

Khanchandani, R. and S. Gillam. 1999. “The Ethnic Minority Linkworker: A Key Member of the Primary Health Care Team?” British Journal of General Practice 49(449): 993-94.

Khandor, E., K. Mason, C. Chambers, K. Rossiter, L. Cowan and S. W. Hwang. 2011. "Access to Primary Health Care among Homeless Adults in Toronto, Canada: Results from the Street Health Survey." Open Medicine 5(2): e94-e103.

Lewin, S., J. Dick, P. Pond, M. Zwarenstein, B. Aja Gvan Wyk and X.P.M. Bosch-Capblanch. 2005. "Lay Health Workers in Primary and Community Health Care" (Review). Cochrane Database of Systematic Reviews (1): CD004015. doi: 10.1002/14651858.

Multicultural Health Brokers Co-Operative (MCHB Co-Op). 2004a. "Articles of Incorporation." (Ed.): 1-5. Edmonton: Author. 
Multicultural Health Brokers Co-Operative (MCHB Co-Op). 2004b. Draft Competency Framework for the Multicultural Health Brokering Practice. Edmonton: Author.

Multicultural Health Brokers Co-Operative (MCHB Co-Op). 2010a."Master List by Broker for Files Ever Open From September 1, 2009 to September 22, 2010." Edmonton: Author.

Multicultural Health Brokers Co-Operative (MCHB Co-Op). 2010b. “Perinatal Health Program Annual Report April 1, 2009 - March 31,2010" Vol. 1. Edmonton: Author.

Meyer, M., S. Torres, N. Cermeño, L. MacLean and R. Monson. 2003. “Immigrant Women Implementing Participatory Research in Health Promotion." The Western Journal of Nursing Research 25(7): 815-34.

Ministry of Health and Long Term Care (MOHLTC). 2008. Ontario Public Health Standards 2008. Toronto: Government of Ontario. Retrieved February 22, 2014. <http://www.health.gov.on.ca/en/pro/programs/publichealth/oph_standards/docs/ophs_2008.pd>.

National Center for Cultural Competence. 2004. Bridging the Cultural Divide in Health Care Settings. The Essential Role of Cultural Broker Programs. Rockville, MD: National Health Service Corps Bureau of Health Professions Health Resources and Services Administration U.S. Department of Health and Human Services (HRSA).

Ortiz, L.M. 2003. "Multicultural Health Brokering: Bridging Cultures to Achieve Equity of Access to Health." $\mathrm{PhD}$, Unpublished Doctoral Dissertation. University of Alberta, Edmonton.

Pottie, K., L. Ortiz and A. Tur Kuile. 2008. "Here's a Thought ... Preparing for Diversity: Improving Preventive Health Care for Immigrants." Our Cities. Ottawa: Metropolis.

Pottie, K., C. Greenaway, J. Feightner, V. Welch, H. Swinkels, M. Rashid et al. 2011."Evidence-Based Clinical Guidelines for Immigrants and Refugees." Canadian Medical Association Journal 1-7. 183(12): E824-925. doi: 10.1503/cmaj.090313.

Rhodes, S.D., K. Long Foley, C.S. Zometa and F.R. Bloom. 2007."Lay Health Advisor Interventions Among Hispanics/Latinos. A Qualitative Systematic Review." American Journal of Preventive Medicine 33(5): 418-27.

Rodrigues Fausto, M.C., L. Giovanella, M.H. Magalhaes de Mendonca, P. Fidelis de Almeida, S. Escorel et al. 2011. "The Work of Community Health Workers in Major Cities in Brazil Mediation, Community Action, and Health Care." Journal of Ambulatory Care Management 34(4): 340-53.

Spitzer, D. 2004. “In Visible Bodies: Minority Women, Nurses, Time, and the New Economy of Care." Medical Anthropology Quarterly 18(4): 490-508.

Stake, R.E. 1995. The Art of Case Study Research. Thousand Oaks, CA: Sage Publications.

Stake, R.E. 2006. "Multiple Case Study Analysis." New York, NY: The Gilford Press.

Swider, S.M. 2002. "Outcome Effectiveness of Community Health Workers: An Integrative Literature Review." Public Health Nursing 19(1): 11-20.

Torres, S. 2013. “Uncovering the Role of Community Health Worker/Lay Health Worker Programs in Addressing Health Equity for Immigrant and Refugee Women in Canada: An Instrumental and Embedded Qualitative Case Study." PhD, Unpublished Doctoral Dissertation. University of Ottawa, Ottawa.

Torres, S., D.L. Spitzer, R. Labonté, C. Amaratunga and C. Andrew. 2013. “Community Health Workers in Canada: Innovative Approaches to Health Promotion Outreach and Community Development among Immigrant and Refugee Populations." Journal of Ambulatory Care Management 36(4): 305-318.

Wells, K.J., J.S. Luque, B. Miladinovic, N. Vargas, Y. Asvat, R.G. Roetzheim and A. Kumar. 2011. “Do Community Health Worker Interventions Improve Rates of Review Screening Mammography in the United States? A Systematic Review." Cancer Epidemiol Biomarkers Prev 20(8): 1580-98. doi: 10.1158/1055-9965.

Yin, R.K. 2003. “Case Study Research Design and Methods” (3rd ed. Vol. 5). Thousand Oaks, CA: Sage Publications. 